\title{
On the Identification and Decomposition of the Unsteady Losses in a Turbine Cascade
}

\author{
D. Lengani* \\ DIME - Universitá di Genova \\ Genova, Italy \\ davide.lengani@edu.unige.it

\section{R. Pichler} \\ University of Melbourne \\ Melbourne, Australia \\ richard.pichler@unimelb.edu.au
}

\section{Michelassi}

Baker Hughes, a GE Company

Firenze, Italy

vittorio.michelassi@ge.com

\author{
D. Simoni
}

DIME - Universitá di Genova

Genova, Italy

daniele.simoni@unige.it

\section{R. Sandberg}

University of Melbourne

Melbourne, Australia

richard.sandberg@unimelb.edu.au

\begin{abstract}
The present paper describes the application of Proper Orthogonal Decomposition (POD) to Large Eddy Simulation (LES) of the T106A low-pressure-turbine profile with unsteady incoming wakes at two different flow conditions. Conventional data analysis applied to time averaged or phase-locked averaged flow fields is not always able to identify and quantify the different sources of losses in the unsteady flow field as they are able to isolate only the deterministic contribution. A newly developed procedure allows such identification of the unsteady loss contribution due to the migration of the incoming wakes, as well as to construct reduced order models able to highlight unsteady losses due to larger and/or smaller flow structures carried by the wakes in the different parts of the blade boundary layers. This enables a designer to identify the dominant modes (i.e. phenomena) responsible for loss, the associated generation mechanism, their dynamics and spatial location.

The procedure applied to the two cases shows that losses in the fore part of the blade suction side are basically unaffected by the flow unsteadiness, irrespective of the reduced frequency and the flow coefficient. On the other hand, in the rear part of the suction side the unsteadiness contributes to losses prevalently due to the finer scale
\end{abstract}


(higher order POD modes) embedded into the bulk of the incoming wake. The main difference between the two cases has been identified by the losses produced in the core flow region, where both the largest scale structures and the finer ones produces turbulence during migration. The decomposition into POD modes allows the quantification of this latter extra losses generated in the core flow region, providing further inputs to the designers for future optimization strategies.

\section{INTRODUCTION}

The aerodynamic efficiency of a turbine blade is a function of the blade geometry, the operating conditions as well as the inlet conditions. Particularly, since the flow within turbomachinery is inherently unsteady, the propagation of the wakes shed from the upstream rows has a large impact on the blade performance. In the last years, accurate experiments (e.g. [1-3]) as well as high-fidelity computations (e.g. [4-6]) have analyzed in detail the unsteady wake migration process. The wake induces a jet-like flow that points towards the blade suction side inducing a periodic phase-dependent structure of the boundary layer.

The wakes migrating within the blade passage alter the profile losses when compared to a steady state case since the wakes excite the suction side boundary layer. At the same time the wake undergoes strong bowing, dilatation, stretching and compression processes within the blade passage and as a consequence, in the so called core flow, turbulence kinetic energy is produced by the shear and strain action of these processes [7]. The losses induced by this mechanism are not negligible and have been the subject of very recent works (e.g. [8-10]).

The wakes are also carrying finer scale turbulent structures, that are forced into the blade boundary layers. These structures are responsible for the generation of a dense population of streaky structures in the footprint of the region perturbed by wakes, as observed both numerically or experimentally (e.g. [3,11-13]); they may further alter the stability of the boundary layers exciting the transition process. An early transition may have a positive impact on the blade efficiency if it suppresses the boundary layer separation, otherwise it is detrimental on the overall performances.

It is clear that the profile losses evaluated under steady conditions are far different than the losses in a real environment (e.g. Michelassi et al. [7]). The different dynamical features that occur within the blade passage affect to different extent the turbine blade efficiency. Losses are produced within all of the passage, by the upstream wake migration, by the turbulence carried by them, and of course by the flow structures developing within the boundary layers. Understanding the flow physics may help the engineers to detect the different sources of losses in order to increase the efficiency of modern new generation turbines. Indeed, while the most recent numerical and experimental techniques may provide a very deep physical insight, tools for the quantification of the different loss sources and their association to particular dynamics have so far not been available. Quantifying these different loss sources may be a further method to observe the impact of the variation of a specific design parameter. For example Michelassi et al. [7,9] used a control volume method [14] on LES and DNS to quantify the entropy generation due to wake migration. Classical post-processing tools, such as phase-averaging [15], cannot be adopted to provide a direct separation and estimation of the sources of unsteady losses.

Recently, Lengani et. al $[10,16]$ identified and evaluated the turbulence kinetic energy production sources by means 
of particle image velocimetry measurements and Proper Orthogonal Decomposition (POD). These works exploited the link between Reynolds stresses and dissipation mechanisms (see for example the work of Moore et al. [17]) and the capability of POD to evaluate the quota to the Reynolds stresses of different dynamics [18]. Even though there is a growing interest in applying POD to turbine blades (e.g. [19-21]), the application provided by Lengani et al. [10] is, at least to the knowledge of the authors, unique since it allows the evaluation of the turbulence kinetic energy produced by the different unsteady flow features, hence accounting separately the loss generation mechanism of different dynamics. However, it has the limit to study only the loss generation due to the production of turbulence kinetic energy.

The present work extends this previous work applying a new post-processing procedure to the high-fidelity LES data of Michelassi et al. [9] simulating a turbine blade under two different unsteady inlet conditions. The new POD based procedure is proposed in order to provide a direct quantification of the entropy generated by each of the different dynamical features that occur within the blade passage. The application of such technique to LES data allows the computation of the most relevant terms that contribute to the rate of change of entropy and not only to the mean flow mechanical energy dissipation as in the previous works. The paper discusses in details the procedure that is based on a Reynolds averaged formulation of the Navier Stokes equations and the application of POD to decompose the Reynolds stresses. The contribution of each POD mode to the entropy rate of change is evaluated and the capability of this procedure to split and quantify the sources of unsteady losses is discussed for two different inlet flow conditions.

\section{LARGE EDDY SIMULATIONS}

The low-pressure turbine data analyzed in the current study were generated using highly resolved large eddy simulations (LES), conducted with the in-house compressible multi-block structured Navier-Stokes solver HiPSTAR. A detailed description of the numerical methods and a rigorous validation for turbomachinery applications is provided in [22]. Here only a brief summary of the key features is presented for completeness. For the discretization of spatial derivatives, fourth-order accurate wavenumber optimized compact finite differences [23] are used in the axial/pitchwise plane, while in the homogeneous spanwise direction a pseudo-spectral approach, relying on the FFTW library, is used. An ultra-low storage five-step explicit fourth-order Runge-Kutta method [24] was used for the time integration. For enhanced robustness of the numerical scheme and to avoid aliasing errors of the cubically nonlinear convective terms within the discretized Navier-Stokes equations, a skew-symmetric splitting of the non-linear terms according to Kennedy et al. [25] was employed.

Using this combination of numerical methods has been shown to accurately reproduce the flow in linear low-pressure turbine cascades [22], and high-pressure turbine cascades [26]. LES models were implemented into the code to reduce computational cost and to allow for parametric studies of turbine flows. The suitability and accuracy of the WALE model [27] was demonstrated in another LPT study that investigated the combined action of reduced frequency and flow coefficient on unsteady loss generation [9]. The numerical set-up for the large-eddy simulations conducted to provide LPT data for the POD analysis follows that described in [7]. A linear low-pressure turbine cascade, featuring the T106A profile, was considered at isentropic conditions of $R e_{2 i s}=100,000$ and $M_{2 i s}=0.4$, based on axial chord and isentropic exit velocity $V_{2 i s}$. The grid was composed of nine blocks, with four blocks for an O-type grid around the blade to ensure optimal grid quality in the 
vicinity of the wall, and five blocks for an H-type grid allowing for pitchwise periodicity. All blocks were connected to each other using characteristic interface conditions in order to ensure high-quality solutions at the block interfaces despite metric discontinuities. At solid walls, no-slip isothermal boundary conditions were used. An immersed boundary method [28] was used to generate periodically incoming bar wakes, placing cylinder bars with diameter $D / C_{a x}=0.02$ at $x / C_{a x}=-0.7$, and moving them in the pitchwise direction with velocity $V_{b a r}$. By varying $V_{b a r}$ the reduced frequency, $F_{r e d}=\frac{f \cdot C_{a x}}{V_{2 i s}}$, was varied, where $f$ is the bar passing frequency.

The data post-processed in this paper are limited to the O-type blocks around the blade surface and one H-type block in the blade passage as represented in Fig. 1. Data are obtained for two different flow conditions (presented in a previous publication [9]): the case 1B1U is defined with $F_{\text {red }}=0.311$ and flow coefficient $\Phi=1.698$, while for the case $1 \mathrm{~B} 3 \mathrm{U} F_{\text {red }}=0.933$ and $\Phi=0.569$. These different conditions of the inflow parameters span the lower and upper limit of current design trend, and generate significantly different incoming wake patterns at the cascade leading edge plane. A more complete view of the design parameter variation is reported in Michelassi et al. [9], where the data have been validated against DNS (see also [22]) and experimental results. Consequently, this data-set serves as a physical test case to check the capability of the POD-based framework to split the different dynamics and quantify their contribution to the entropy rate of change.

\section{Data analysis}

The LES data have been analyzed by means of proper orthogonal decomposition in order to highlight the dynamics related to the different sources of unsteadiness. The method of snapshots of Sirovich [29] has been employed to compute the POD. The POD kernel has been defined on the fluctuating velocity components following the Reynolds decomposition. The eigenvalues of the POD modes therefore represent the turbulence kinetic energy of each mode and the modes are ranked by their energy content. The POD analysis provides two further pieces of information, the eigenvectors $\chi^{(k)}$ and the POD modes $\phi^{(k)}$. Both of these quantities generate a complete orthogonal basis, that retains the temporal information of each mode. The eigenvector can be used to identify the frequency of each POD mode that provides the spatial information in the flow field. It will be shown that this spatio and temporal information can be used to identify groups or reduced order representations of the different dynamics that occur in a complex unsteady flow environment.

A key property of POD is the fact that both modes and temporal eigenvetors form an orthogonal basis. This property implies that the Reynolds shear and normal stresses can be computed, adopting an index notation, as (e.g. [30]):

$$
\overline{u_{i}^{\prime} u_{j}^{\prime}}=\sum_{k} \phi_{u_{i}}^{(k)} \phi_{u_{j}}^{(k)}
$$

The term $\phi_{u_{i}}^{(k)} \phi_{u_{j}}^{(k)}$ represents the contribution of the $k^{t h}$ POD mode to the overall time-mean Reynolds stresses.

Furthermore, since the temporal eigenvectors are a full orthogonal basis, the POD mode of different flow properties, such as, for example, the total enthalpy, may be obtained by projecting the instantaneous enthalpy fields $h_{t}^{\prime}$ onto the temporal 
eigenvector of the velocity correlation tensor, like the extended POD procedure proposed by [31]:

$$
\phi_{h_{t}}^{(k)}=\sum_{n=1}^{N} \chi_{n}^{(k)} h_{t n}^{\prime}
$$

where $n$ is the discrete time interval. These properties will be further exploited in the following two subsections.

\section{Fundamental equations}

The post-processing methodology adopted in the present paper is based on the capability of POD of representing the flow field on a modal basis, see also Lengani et al. [32] for more details. In order to understand the contribution of each unsteady dynamical feature to the loss production rate, the Reynolds averaged Navier-Stokes equations are considered. Arranging these equations, the balance of the time mean total enthalpy $\left(\bar{h}_{t}\right)$ and total pressure $\left(\bar{p}_{t}\right)$ may be written as follows (see $[33,34])$ :

$$
\begin{gathered}
\frac{D \bar{h}_{t}}{D t}=\frac{\partial}{\partial x_{i}}\left[\kappa\left(\frac{\partial \bar{h}_{t}}{\partial x_{i}}\right)-\overline{u_{i}^{\prime} h_{t}^{\prime}}\right]+v \overline{\left(\frac{\partial u_{i}^{\prime}}{\partial x_{j}}+\frac{\partial u_{j}^{\prime}}{\partial x_{i}}\right) \frac{\partial u_{i}^{\prime}}{\partial x_{j}}} \\
\frac{D \bar{p}_{t}}{D t}=-\rho \overline{u_{i}^{\prime} u_{j}^{\prime}} \frac{\partial U_{i}}{\partial x_{j}}-\rho v\left(\frac{\partial U_{i}}{\partial x_{j}}\right)^{2}+\rho v \frac{\partial^{2} K}{\partial x_{j}^{2}}-\frac{\partial\left(U_{i} \overline{u_{i}^{\prime} u_{j}^{\prime}}\right)}{\partial x_{j}}
\end{gathered}
$$

where $\kappa$ is the thermal conductivity, while, $K$ is the kinetic energy of the mean flow. Equations 3 and 4 are then written in non-dimensional form, neglecting the diffusive terms [32]:

$$
\begin{gathered}
\frac{D \bar{h}_{t}}{D t}=\underbrace{-\frac{\partial \overline{u_{i}^{\prime} h_{t}^{\prime}}}{\partial x_{i}}}_{I}+\underbrace{\frac{1}{R e_{\infty}} \overline{\left(\frac{\partial u_{i}^{\prime}}{\partial x_{j}}+\frac{\partial u_{j}^{\prime}}{\partial x_{i}}\right) \frac{\partial u_{i}^{\prime}}{\partial x_{j}}}}_{I I} \\
\frac{D \bar{p}_{t}}{D t}=-\underbrace{\rho \overline{u_{i}^{\prime} u_{j}^{\prime}} \frac{\partial U_{i}}{\partial x_{j}}}_{I I I}-\underbrace{\frac{\rho}{R e_{\infty}}\left(\frac{\partial U_{i}}{\partial x_{j}}\right)^{2}}_{I V}
\end{gathered}
$$


These two terms may be related, by means of Gibbs' equation, to the entropy variation $(D \bar{s} / D t)$ :

$$
T_{t} \frac{D \bar{s}}{D t}=\frac{D \bar{h}_{t}}{D t}-\frac{1}{\rho_{t}} \frac{D \bar{p}_{t}}{D t}
$$

Equations 5 and 6 are substituted in eq. 7 in order to obtain the balance equation over a control volume covering the cascade passage:

$$
\tilde{s}_{2}-\tilde{s}_{1}=\frac{1}{\dot{m}}\left(\int_{V} \frac{(I+I I)}{T_{t}} d V-\int_{V} \frac{(I I I+I V)}{T_{t} \rho_{t}} d V\right)
$$

the terms $\tilde{s}_{i}$ denotes the inlet and outlet mass-averaged cascade entropy per unit mass. The term $I$ on the right hand side provides the entropy rate of change due to the enthalpy-velocity correlation terms and the term $I I$ provides the fine (resolved) turbulent scale dissipation producing heat. The term III represents the mechanical energy dissipation due to the turbulent tresses, while the term $I V$ represents the viscous dissipation. As previously discussed, the diffusive terms of Eqs. 3 and 4 are neglected since their volume integral values are about zero.

By means of this approach, the entropy variations are related to the terms I, II, III, and IV of equation 8 . Since the first three terms are due to the turbulence activity, being defined by fluctuating quantities according to the Reynolds decomposition, the contribution to the entropy rate of change of each POD mode can be computed. Namely, since the POD modes are a statistical representation of the different dynamics, a quantitative and local estimation of the entropy rate of change of the different dynamics can be computed by means of this method. Only the viscous dissipation represented by term IV of Eq. 8 cannot be decomposed since it is not dependent on the dynamics. This term is accounted in the cumulative distributions of losses since it represents a large part of the overall losses.

\section{DATA PROCESSING}

The computation of terms I, II, and III is discussed in this section making use of the properties of POD that have been previously introduced (see equations 1 and 2 and Lengani et al. [32]).

The term III of eq. 8 is introduced at first. The TKE production rate $p_{i j}$ can be written as a function of the contribution of each POD mode to the Reynolds stress $\left(\phi_{u_{i}}^{(k)} \phi_{u_{j}}^{(k)}\right.$ according to eq. 1):

$$
p_{i j}^{(k)}=-\phi_{u_{i}}^{(k)} \phi_{u_{j}}^{(k)} \frac{\partial U_{i}}{\partial x_{j}}, \quad \text { with } \quad p_{i j}=\sum_{k} p_{i j}^{(k)}
$$

where each $\mathrm{k}^{\text {th }}$ POD mode contributes to a quota of the time-mean TKE generation rate (as also discussed previously in Lengani et al. [16,32]). 
The term II of eq. 8, representing the dissipation of energy due to the resolved scales, can be computed as a combination of POD modes of the velocity field and their spatial derivatives (see also [35]). It is expressed as:

$$
\frac{1}{R e_{\infty}} \overline{\sigma_{i, j} \frac{\partial u_{i}^{\prime}}{\partial x_{j}}}=\frac{1}{R e_{\infty}} \sum_{k}\left(\frac{\partial \phi_{i}^{(k)}}{\partial x_{j}}+\frac{\partial \phi_{j}^{(k)}}{\partial x_{i}}\right) \frac{\partial \phi_{i}^{(k)}}{\partial x_{j}}
$$

The term I of Eq. 8, representing the enthalpy-velocity correlation term, can be expressed as:

$$
-\frac{\partial \overline{u_{i}^{\prime} h_{t}^{\prime}}}{\partial x_{i}}=-\sum_{k} \frac{\partial \phi_{u_{i}}^{(k)} \phi_{h_{t}}^{(k)}}{\partial x_{i}}
$$

where the POD mode of the enthalpy $\phi_{h_{t}}^{(k)}$ is computed according to eq. 2.

Lengani et al. [32] discussed the convergence and error of this procedure. Namely, it has been discussed that the relative error in the computation of the TKE captured by the first 30, most energetic modes, is below $0.5 \%$. The error on the higher order modes is minimized summing up their contribution to the entropy rate of change when considering ensembles of modes that correspond to different dynamics.

\section{RESULTS AND DISCUSSION}

\section{Phase-averaged flow-field}

The phase averaged flow fields are presented at first in order to introduce the two different flow conditions. They are referred to as 1B1U, high flow coefficient and small reduced frequency case, and as 1B3U, lower flow coefficient and higher reduced frequency, as first introduced in Michelassi et al. [9]. Although the phase-averaged flow fields have been already discussed in detail in this previous paper it seems appropriate to provide here a brief summary of the main differences between these two cases. Three snapshots of the phase averaged flow field for each case are represented in Fig. 2, the case 1B1U on top while 1B3U is on bottom (as it will be also for all the following pictures). The time interval between snapshots is one third of the wake passing period and the first snapshot identifies the wake entering into the blade passage. The contour plots represent the phase averaged distribution of the resolved turbulence kinetic energy, while the superimposed vectors show the perturbation velocity field defined as the difference between the phase averaged velocity field and the time mean one.

The wake can be identified by the perturbation velocity vectors that point towards the suction side, this flow structure is surrounded by two counter-rotating vortices. This is the vectorial representation of the wake induced jet that points toward the wall and opens into two branches. The first, counter-clockwise rotating vortex is at the leading boundaries of the wake and is often called "Q1" (e.g. [36]) and the second one is clockwise rotating and is called "Q2". This structure can be observed in the different snapshots for both flow conditions. It may be also observed that, as also pointed out by other works (e.g. [3]), the largest TKE activity is found at the leading boundaries of the wake, namely between the Q1 vortex and the 
jet-like effect.

The two cases present a different scenario, the 1B1U case is characterized by a low reduced frequency and hence just one wake at a time can be observed within the blade passage. The reduced frequency of the $1 \mathrm{~B} 3 \mathrm{U}$ case is higher and then the passage is excited by about two wakes at a time (e.g. in the first snapshot on bottom of Fig. 2 a wake is entering the passage and another one is leaving the passage). Furthermore, the wake path at the cascade inlet plane becomes increasingly tangential with decreasing flow coefficient. Moreover, also the large scale vortices (Q1 and Q2) attached to the wake patch during migration appear with smaller dimension for the 1B3U case, and consequently induce a significantly smaller distortion (see the perturbation vectors) during migration.

Different distributions of the turbulence kinetic energy within the blade passage can also be observed. The 1B1U case shows that the TKE within the blade passage is almost zero before the wake enters, then the TKE reaches its maximum values in proximity of the suction side. For the 1B3U case, the TKE remains at high values within the whole blade passage since more than one wake is present at the same time, there is just a small region at minimum TKE between one wake and the following one (this region is defined by the perturbation velocity vectors pointing towards the pressure side). For this latter case, the TKE activity on the pressure side is also considerably higher than that of the former.

However, the phase averaged TKE provides just a statistical representation of the turbulence carried by the wake, while the perturbation velocity vectors account for the deterministic fluctuating components of the velocity vector that are periodically induced at each cycle of the passing wake. The purpose of the next section is to discuss the same quantities with a different representation based on a kinematic kernel and through orthogonal functions.

\section{POD analysis}

As discussed in a recent publication [10], the POD can be used to identify different dynamical features that occur within a blade passage with incoming passing wake. The velocity perturbations are no more divided into two families (deterministic and stochastic) but are represented by POD modes, ranked by the overall TKE, each of which captures a percentage of the Reynolds stress tensor.

The energy rank is the first information provided by the POD eigenvalues $\lambda$ that quantifies the kinetic energy captured by the POD modes and their relative importance. Figure 3 shows the cumulative contribution of each POD eigenvalue normalized with the total kinetic energy of the 1B3U case. Note that the abscissa is in log scale since the contribution of each POD mode becomes lower going towards higher order modes and they provide the statistical representation of the smaller scale structures.

The energy of the low order POD modes of the 1B1U case is higher than in the 1B3U case. Hence, the POD highlights that the largest scale structures of this case posses a higher energy when compared to that of the 1B3U case, consistent with the larger perturbation velocity vectors previously observed for this condition. Indeed, it will be confirmed in the following, that the first POD modes are representative of the deterministic velocity perturbation induced by the wake (as also observed in previous papers, e.g. [10]). This is consistent with the phase averaged results of Fig. 2, where the perturbation velocity vectors, that represent the deterministic fluctuations, have a larger magnitude for the 1B1U case. Increasing the order of 
the mode, the energy captured by each mode for the 1B3U case becomes larger: the consecutive wakes of this case carry more turbulence that the other case (as also observed in Michelassi et al. [9]). This is coherent with the phase-averaged results. However, each higher order POD mode provides the description of the turbulence carried by the wakes with a modal decomposition, while the phase-averaged TKE provides only their statistical representation.

The analysis of the temporal information (the POD eigenvectors) further supports the previous observations and allows more clearly the distinction of the different dynamics. As an example, the spectral analysis of the eigenvectors of the first four modes is represented in Fig. 4. This analysis confirms that the first two modes occur at the wake passing frequency, identifiable as a sharp peak in the spectrum at the fundamental frequency. For case 1B1U, the third and fourth mode are related to the harmonic of the wake passing frequency. Hence, for this case the first four modes capture the same dynamics, namely the periodic passing of the wake. For case 1B3U, the third and fourth mode are instead representing a different dynamic that occurs at frequency lower than that of the wake passing.

The spatial distribution of these modes is shown in Fig. 5 as vectorial representation, where each vector component is provided by the POD mode of the corresponding velocity component. The contour plot shows the normalized TKE captured by the mode. A 2D representation is provided for clarity, whereas modes are 3D as obtained from the original instantaneous dataset.

The first and second POD modes depict the same flow structure identifying large scale vortices that represents the “jet-like" wake effect $[3,6]$. These two modes have the same energy and the same frequency albeit spatially shifted. This behaviour is typical for pseudo-periodic convective flows [37-40]. The deterministic part of the wake migrating across the blade passage may be reproduced accounting for these and similar POD modes. For the case 1B1U, the third and fourth modes also contribute to the deterministic part of the velocity fluctuations and should be used together with the previous two modes to properly represent the deterministic fluctuations. For the other case, modes three and four are no more related to the deterministic passing wake events according to the spectral analysis, while it seems related to a low frequency motion affecting just the rear part of the blade pressure side where typically Görtler vortices are present (e.g. [4]). Consistently to what was previously shown, the characteristic dimension of the structures highlighted by modes 1 and 2 for the 1 B3U case are evidently smaller than in the 1B1U case. The procedure thus provides a modal representation of the response of the flow distortion into the core flow to the variation of the flow parameters.

It is worth noting that a single POD mode is not able to completely describe a structure or a particular dynamics. As an example the first four modes of the case 1B1U are certainly needed together to provide the representation of the deterministic velocity fluctuations of the wake. An ensemble of POD modes that pertains and describes the same dynamical feature is needed to describe the losses produced by that dynamics. On the basis of the information provided by the POD it is possible to define a series of different ensembles of POD modes (i.e., reduced order models) accounting for different physical effects. For simplicity, three families of modes have been defined and adopted in the present paper. The first ensemble contains all the modes related to the deterministic passing wakes, this ensemble can be accurately chosen by means of spectral analysis including just the modes at the harmonics of the wake passing frequency. The two further ensembles are instead defined on the basis of an energy based criteria. The second ensemble contains modes up to mode 300, while the last family contains 
the remaining modes in order to capture less than $20 \%$ of the TKE and represents the finer scale structures of turbulence. Representing the different dynamical features as ensembles of modes minimize the bias error related to the choice of specific modes. This criteria is here adopted to minimize this error, but it has to be noted that the number of dynamical features extracted from POD can be higher supposed that the different features can be properly identified and isolated with different criteria.

\section{TKE production}

The spatial distribution of the rate of change of entropy due to turbulence kinetic energy production (term III of eq. 8) is reported in the present section separating the loss contribution of the three families of POD modes described above. The $2 \mathrm{D}$ view is presented in Fig. 6 for the two cases. The plot on the left represents the loss generation due to the large scale structures of the wake during migration in the downstream passage. For both cases the largest TKE production occurs within the passage upstream of the peak suction position, as well as in the aft portion of the suction side. The loss generation in the blade passage upstream of the peak suction is identified in the region where the wake is dilated and bowed. The losses in the rear suction side are produced by the development of the boundary layer and its interaction with the large and small scale vortical structures caused by the migrating wake. However, the two cases show significant differences, the TKE production for the case 1B1U shows a larger region than the 1B3U case where losses are produced (blue area), this is consistent with the evidently larger contribution of the wake related modes to the Reynolds shear stresses (represented by the vectors in Fig. 5) previously discussed. This plot also highlights zones where the turbulence production is negative. This phenomenon is called back-scatter and represents a local flux of energy from small to large scale structures that partially compensate the production of turbulence (see also Doering and Gibbon [41] for the simple case of a 2D inviscid flow). Overall, the volume integral of the energy flux, that will be provided in the next section, is positive. The region at "negative production" is also larger and closer to the pressure side for the 1B1U than for the 1B3U case, for which back-scatter mainly occurs in the stagnation region.

The plot in the middle represents the mid range modes: TKE production occurs in the pressure and suction side boundary layers but also within the blade passage. In this latter region the losses are caused by the small scale turbulence structures carried by the wakes. This source of losses was rarely considered in previous studies, and started to be appreciated only in recent publications (e.g. [8-10]). The effect of the turbulence carried by the wake on the TKE and hence loss production can be better appreciated when comparing the two cases. The case 1B3U, for which the wakes are very close and generate turbulence, has very high losses within the blade passage. On the other hand, for the 1B1U case the smaller turbulence activity within the wake (as seen in Fig. 2) produces evidently smaller TKE just close to the suction side where the wake is transported.

The losses isolated by the POD modes related to the finer scale structures (right column of Fig.6) occur mainly in the decelerating part of the blade suction side. The high level of TKE production observed for this ensemble of POD modes is induced by the smaller scale, less energetic, and stochastic structures that grow into the boundary layer in the rear suction side. Since the results of the two cases are almost identical it seems that the TKE production mechanism due to the finer 
scale structures is poorly related to wake migration within the channel.

\section{Contribution to losses}

The contribution of the TKE production is the dominant term to the entropy rate of change when compared with the other three terms (see eq. 8), since it is responsible for more than $50 \%$ of the overall entropy rate of change. However, the total production of losses is comprised by the four terms of eq. 8. In order to provide the total losses, the four terms of eq. 8 have been integrated over the whole 3D cascade computational domain, accounting for the contribution of each mode. The viscous dissipation term (term IV of eq. 8), is the second highest contribution (about 35\% of the total) but it is not decomposed by modes as previously explained. This contribution is represented in the following pictures with cross symbols as a base level for the cumulative contribution to losses.

The rate of change of entropy has been integrated in different flow regions, as sketched in Fig. 1: the boundary layer region, the potential flow region of the blade passage, and the mixing region downstream of the trailing edge. The distinction between boundary layer and core flow region has been obtained by a vorticity threshold criteria [32]. The mixing region has been defined by a line normal to the flow direction downstream of the trailing edge.

The purpose of this procedure is the identification of where, in space, the dynamical features (the reduced order model defined by an ensemble of POD modes) act to produce losses. The results are shown in Fig. 7 as cumulative contributions to the losses (entropy rate of change) of each POD mode. The black line represents the losses produced in the whole computational domain considered for this analysis (as shown Fig. 1). Two dashed vertical lines are superimposed to the plot to distinguish between the different ensembles of POD modes as defined in the previous section. Results are normalized by the total losses of the $1 \mathrm{~B} 3 \mathrm{U}$ case.

Since the plots represent a cumulative contribution, the last mode shows the total amount of losses within the whole blade passage. The 1B3U case shows that losses generated within the blade core flow region (grey line) are almost the same as those in the boundary layer (red line). Indeed, the main difference between the losses of the two cases is the amount of losses produced in the core flow.

The new insight that this loss estimation procedure enables is the capability to look for which dynamical features are responsible for the different losses produced. In this case, looking at the contribution of each family of POD modes it is possible to observe that the main difference between the two cases consists in the loss produced in the core flow region by the mid range modes. In this region, the losses due to the wake related modes, that have been identified by means of the Fourier analysis of the POD eigenvector, are similar for the two cases and of the order of 10\%. For the case 1B3U the graph should be read excluding modes 3 and 4 that are not related to the harmonics of the wake passing frequency, as previously observed. This contribution is slightly higher for the 1B1U case since the deterministic perturbation induced by the wake in this case is larger, as observed from the distribution of the POD eigenvalues and from phase-averaged results. The losses of the $1 \mathrm{~B} 3 \mathrm{U}$ case start to abruptly increase above mode 10 . These losses are related to modes that represent the turbulence carried by the wake, that contributes about $26 \%$ of the total, while for the case $1 \mathrm{~B} 1 \mathrm{U}$ their contribution is about $10 \%$. Hence, The finer scale structures of the turbulence carried by the wake in the core flow, modes above 300 , account for about $7 \%$ of 
the total and are similar for both cases.

The losses in the boundary layer are mainly produced by the viscous dissipation, that is represented as a base level and corresponds to about $28 \%$ of the total for both cases. Looking at the trend of the red line it may be observed that the large scale structures related to the wake migration produce just a small, almost negligible, amount of losses into the boundary layer region. The smaller scale structures, represented by the modes above 50 , provide $25 \%$ of the total losses. They constitute the remaining quota of losses generated within the boundary layer and are about equally split between the dynamics identified by the "mid range" and "higher order" POD modes. This means that the wake boundary layer interaction process is mainly dominated, at least in terms of loss production, by the turbulence embedded into the wake. The remainder of the loss budget is generated in the mixing region. Also in this case the largest scale dynamics, the most energetic POD modes, do not contribute to the loss generation in this region, and the variation of the flow parameters provides negligible effects.

A further analysis step is provided in Fig. 8. There, the losses in the boundary layer region are analyzed in detail splitting their contribution to four zones: the stagnation region around the leading edge of the blade, the pressure side, and the suction side is separated into two regions: the accelerating and decelerating ones. The total amount of losses in the boundary layer is shown as a reference with a solid red line. The largest amount of losses produced by the dynamical features is limited to the decelerating part of the boundary layer, while for the rest the largest contribution is captured by the viscous dissipation (base level).

Losses in the accelerating part of the suction side boundary layer are equal for both cases and only depends on the viscous dissipation (the lines are horizontal). This means that the wake-boundary layer interaction is negligible in this flow region, both for the deterministic large-scale and finer structures carried by the wakes. The main differences between the two cases are observed in the pressure side and in the decelerating part of the boundary layer. In this latter region, the larger amount of turbulence carried by the wakes (mid range and higher order modes) for the 1B3U case induces larger losses than for the other case.

The pressure side boundary layers also show different loss behaviour between the two cases. For the 1B1U case, the cumulative loss distribution is dominated by the viscous losses. For the 1B3U case, the mid range modes generate losses. The higher turbulence level carried by the wakes for this latter case is inducing the formation of coherent structures within the boundary layer, probably driving it to an advanced transitional state with breakdown of boundary layer streaks, as also observed in the previous publication of Michelassi et. al [9].

\section{CONCLUSIONS}

Proper orthogonal decomposition has been applied to high fidelity LES data of a low pressure turbine blade with different unsteady inflow conditions. A new post-processing procedure, that makes use of the properties of POD, has been described and applied in order to identify and quantify how the different dynamical features affect the losses. It has been shown that POD can provide a representation of the unsteady flow features that complete the information provided by classical analysis techniques such as time and phase averaging of the data. POD describes the dynamical features found in the unsteady flow 
field by ensembles of properly selected modes that constitute reduced order models of the flow field. This scale separation can be used to describe losses due to migration of large scale structures into the core flow, as well as that due to the finer scale structures in the different parts of the blade boundary layers. Such reduced order models enable a much more focused analysis that can guide a designer towards unsteady loss reduction.

For the two flow fields under scrutiny here, cases 1B1U $\left(\Phi=1.698\right.$ and $\left.F_{\text {red }}=0.311\right)$ and $1 \mathrm{~B} 3 \mathrm{U}(\Phi=0.569$ and $\left.F_{\text {red }}=0.933\right)$, losses in the accelerating part of the blade suction side are essentially due to viscous effects, with negligible contribution due to unsteadiness sources (neither large nor finer scale structures carried by the wake). Otherwise, on the rear suction, the unsteady boundary layer losses are mainly driven by the mid range modes, representative of structures carried by the wake into the boundary layer. Losses in the mixing region are not affected by the large scale structures of the wake, and appear hardly influenced by the parameters variation. The main difference between the two cases has been identified in the losses generated in the core flow region. The case 1B3U, with lower flow coefficient and higher reduced frequency, is in fact characterized by high values of TKE within the whole blade passage since more than one wake is present at the same time.

The proposed post-processing procedure is able to determine exactly the flow structures and features responsible for the predicted loss shift between the two cases. POD allows a deeper analysis into the associated loss generation, as it points to the extra losses in the core flow region that are caused in this case by the large-scale turbulence carried by the incoming wakes. Particularly, this analysis procedure provides a modal representation of the response of the flow field and its entropy rate of change to the variation of the flow parameters. Hence, it may be adopted to provide a fast analysis of the impact of variations of design parameters on expected losses with a great amount of details, since the sources of losses are isolated in space and classified for the different dynamical features.

\section{ACKNOWLEDGMENT}

The authors would like to thank General Electric Global Research for permission to publish this work. Computations have been conducted on the UK national facility ARCHER using resources provided by the UK turbulence consortium under Grant No. EP/L000261/1.

\section{References}

[1] Stieger, R. D., and Hodson, H. P., 2005. "The unsteady development of a turbulent wake through a downstream low-pressure turbine blade passage". Journal of Turbomachinery, 127, pp. 388-394.

[2] Hodson, H. P., and Howell, R. J., 2005. "Bladerow interactions, transition, and high-lift aerofoils in low-pressure turbines". Annu. Rev. Fluid Mech., 37, pp. 71-98.

[3] Lengani, D., Simoni, D., Ubaldi, M., Zunino, P., and Bertini, F., 2016. “Coherent structures formation during wakeboundary layer interaction on a lp turbine blade". Flow, Turbulence and Combustion, pp. 1-25.

[4] Wu, X., Jacobs, R., Hunt, J. C. R., and Durbin, P. A., 2001. "Evidence of longitudinal vortices evolved from distorted wakes in a turbine passage". J. Fluid Mech., 446, pp. 199-228. 
[5] Michelassi, V., Wissink, J. G., Fröhlich, J., and Rodi, W., 2003. "Large-eddy simulation of flow around low-pressure turbine blade with incoming wakes”. AIAA journal, 41(11), pp. 2143-2156.

[6] Sarkar, S., and Voke, P., 2006. "Large-eddy simulation of unsteady surface pressure overa low-pressure turbine blade due to interactions of passing wakes and inflexional boundary layer”. Journal of Turbomachinery, 128, pp. $221-231$.

[7] Michelassi, V., Chen, L.-W., Pichler, R., and Sandberg, R. D., 2015. "Compressible direct numerical simulation of low-pressure turbines-part II: Effect of inflow disturbances”. Journal of Turbomachinery, 137(7), pp. 071005 1-12.

[8] Praisner, T., Clark, J., Nash, T., Rice, M., and Grover, E., 2006. "Performance impacts due to wake mixing in axial-flow turbomachinery”. In ASME Turbo Expo 2006, Barcelona, Spain, May 8-11, Paper No. GT2006-90666.

[9] Michelassi, V., Chen, L., Pichler, R., Sandberg, R., and Bhaskaran, R., 2016. "High-fidelity simulations of low-pressure turbines: Effect of flow coefficient and reduced frequency on losses". Journal of Turbomachinery, 138(11), p. 111006.

[10] Lengani, D., Simoni, D., Ubaldi, M., Zunino, P., Bertini, F., and Michelassi, V., 2017. “Accurate estimation of profile losses and analysis of loss generation mechanisms in a turbine cascade”. Journal of Turbomachinery, 139(12), p. 121007.

[11] Wu, X., Jacobs, R. G., Hunt, J. C. R., and Durbin, P. A., 1999. "Simulation of boundary layer transition induced by periodically passing wakes". Journal of Fluid Mechanics, 398, pp. 109-153.

[12] Nagabhushana Rao, V., Tucker, P., Jefferson-Loveday, R., and Coull, J., 2013. "Large eddy simulations in low-pressure turbines: Effect of wakes at elevated free-stream turbulence”. Int. J. Heat Fluid Flow, 43, pp. 85-95.

[13] Coull, J. D., and Hodson, H. P., 2011. "Unsteady boundary-layer transition in low-pressure turbines". J. Fluid Mech., 681, pp. 370-410.

[14] Denton, J. D., 1993. “Loss mechanisms in turbomachines”. Journal of Turbomachinery, 115(4), pp. 621-656.

[15] Hussain, A., and Reynolds, W., 1970. "The mechanics of an organized wave in turbulent shear flow". J. Fluid Mech., 41, pp. 241-258.

[16] Lengani, D., Simoni, D., Ubaldi, M., Zunino, P., and Bertini, F., 2017. “Analysis of the reynolds stress component production in a laminar separation bubble”. International Journal of Heat and Fluid Flow, 64, pp. 112-119.

[17] Moore, J., Shaffer, D., and Moore, J., 1987. "Reynolds stresses and dissipation mechanisms downstream of a turbine cascade". Journal of Turbomachinery, 109(2), pp. 258-267.

[18] Perrin, R., Braza, M., Cid, E., Cazin, S., Barthet, A., Sevrain, A., Mockett, C., and Thiele, F., 2007. “Obtaining phase averaged turbulence properties in the near wake of a circular cylinder at high reynolds number using POD". Experiments in Fluids, 43(2-3), pp. 341-355.

[19] Sarkar, S., 2008. "Identification of flow structures on a LP turbine blade due to periodic passing wakes". J. Fluid Eng - T ASME, 130, p. 061103 (10 pages).

[20] Romero Martinez, S. R., and Gross, A., 2017. "Numerical investigation of low reynolds number flow in turbine passage”. In 55th AIAA Aerospace Sciences Meeting, Grapevine, Texas, 9-13 January, p. 1456.

[21] Gross, A., Marks, C., and Sondergaard, R., 2017. "Numerical simulations of active flow control for highly loaded low-pressure turbine cascade”. In 55th AIAA Aerospace Sciences Meeting, Grapevine, Texas, 9-13 January, p. 1460. 
[22] Sandberg, R. D., Michelassi, V., Pichler, R., Chen, L., and Johnstone, R., 2015. “Compressible direct numerical simulation of low-pressure turbines—part I: Methodology”. Journal of Turbomachinery, 137(5), p. 051011.

[23] Kim, J. W., and Sandberg, R. D., 2012. "Efficient parallel computing with a compact finite difference scheme". Computers \& Fluids, 58, pp. 70-87.

[24] Kennedy, C. A., Carpenter, M. H., and Lewis, R. M., 2000. "Low-storage, explicit runge-kutta schemes for the compressible navier-stokes equations". Applied numerical mathematics, 35(3), pp. 177-219.

[25] Kennedy, C. A., and Gruber, A., 2008. "Reduced aliasing formulations of the convective terms within the navier-stokes equations for a compressible fluid". Journal of Computational Physics, 227(3), pp. 1676-1700.

[26] Wheeler, A. P. S., Sandberg, R. D., Sandham, N. D., Pichler, R., Michelassi, V., and Laskowski, G., 2016. “Direct numerical simulations of a high-pressure turbine vane”. Journal of Turbomachinery, 138(7), p. 071003.

[27] Nicoud, F., and Ducros, F., 1999. "Subgrid-scale stress modelling based on the square of the velocity gradient tensor". Flow, turbulence and Combustion, 62(3), pp. 183-200.

[28] Schlanderer, S. C., Weymouth, G. D., and Sandberg, R. D., 2017. “The boundary data immersion method for compressible flows with application to aeroacoustics". Journal of Computational Physics, 333, pp. 440-461.

[29] Sirovich, L., 1987. “Turbulence and the dynamics of coherent structures. part I-III”. Q Appl Math, 45, pp. 561-590.

[30] Perrin, R., Cid, E., Cazin, S., Sevrain, A., Braza, M., Moradei, F., and Harran, G., 2007. "Phase-averaged measurements of the turbulence properties in the near wake of a circular cylinder at high reynolds number by 2C-PIV and 3C-PIV". Exp Fluids, 42, pp. 93-109.

[31] Borée, J., 2003. "Extended proper orthogonal decomposition: a tool to analyse correlated events in turbulent flows". Experiments in fluids, 35(2), pp. 188-192.

[32] Lengani, D., Simoni, D., Pichler, R., Sandberg, R., Michelassi, V., and Bertini, F., 2018. "Identification and quantification of losses in a LPT cascade by POD applied to LES data”. International Journal of Heat and Fluid Flow, 70, pp. $28-40$.

[33] Hanjalić, K., and Launder, B., 2011. Modelling turbulence in engineering and the environment: second-moment routes to closure. Cambridge university press.

[34] Leschziner, M., 2015. Statistical turbulence modelling for fluid dynamics—demystified: an introductory text for graduate engineering students. World Scientific.

[35] Lengani, D., Simoni, D., Ubaldi, M., Zunino, P., and Guida, R., 2016. “Turbulence production, dissipation and length scales in laminar separation bubbles". ETMM11, Palermo, Italy, 21-23 Sept, pp. 1-6.

[36] Gompertz, K. A., and Bons, J. P., 2011. "Combined unsteady wakes and active flow control on a low-pressure turbine airfoil”. AIAA Journal of Propulsion and Power, 27, pp. 990-1000.

[37] Legrand, M., Nogueira, J., and Lecuona, A., 2011. "Flow temporal reconstruction from non-time-resolved data part I: mathematic fundamentals". Exp. Fluids, 51(4), pp. 1047-1055.

[38] Legrand, M., Nogueira, J., Tachibana, S., Lecuona, A., and Nauri, S., 2011. "Flow temporal reconstruction from nontime-resolved data part II: practical implementation, methodology validation, and applications”. Exp. Fluids, 51(4), 
pp. 861-870.

[39] Yarusevych, S., and Kotsonis, M., 2017. "Effect of local DBD plasma actuation on transition in a laminar separation bubble". Flow, Turbulence and Combustion, 98(1), pp. 195-216.

[40] Wen, X., Tang, H., and Duan, F., 2016. "Interaction of in-line twin synthetic jets with a separated flow". Physics of Fluids, 28(4), p. 043602.

[41] Doering, C. R., and Gibbon, J. D., 1995. Applied analysis of the Navier-Stokes equations, Vol. 12. Cambridge University Press.

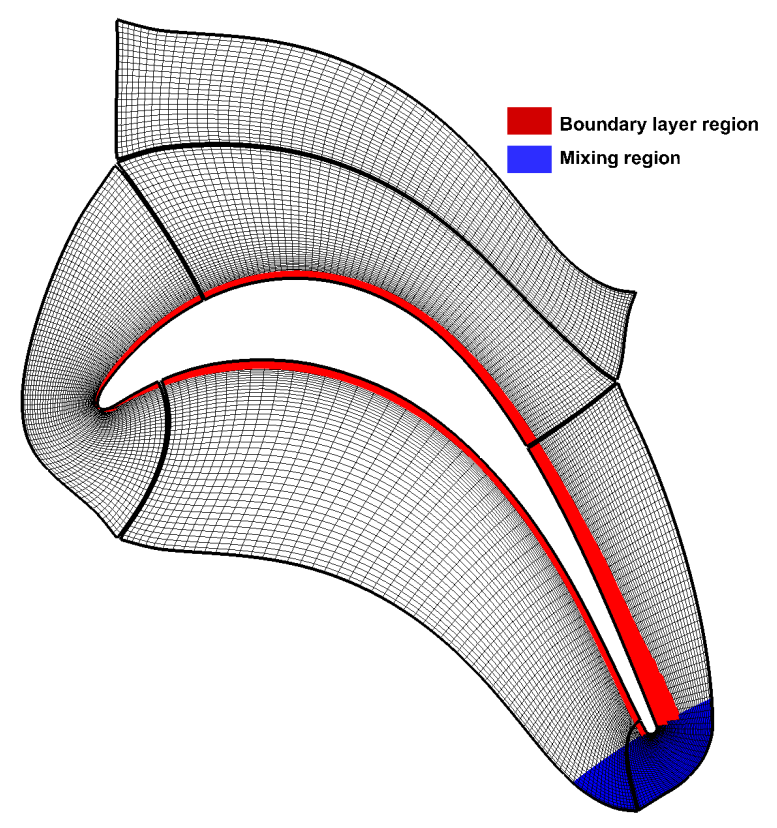

Fig. 1: SCHEME OF THE COMPUTATIONAL DOMAIN ADOPTED FOR THE POST-PROCESSING AND INTEGRATION AREAS. 

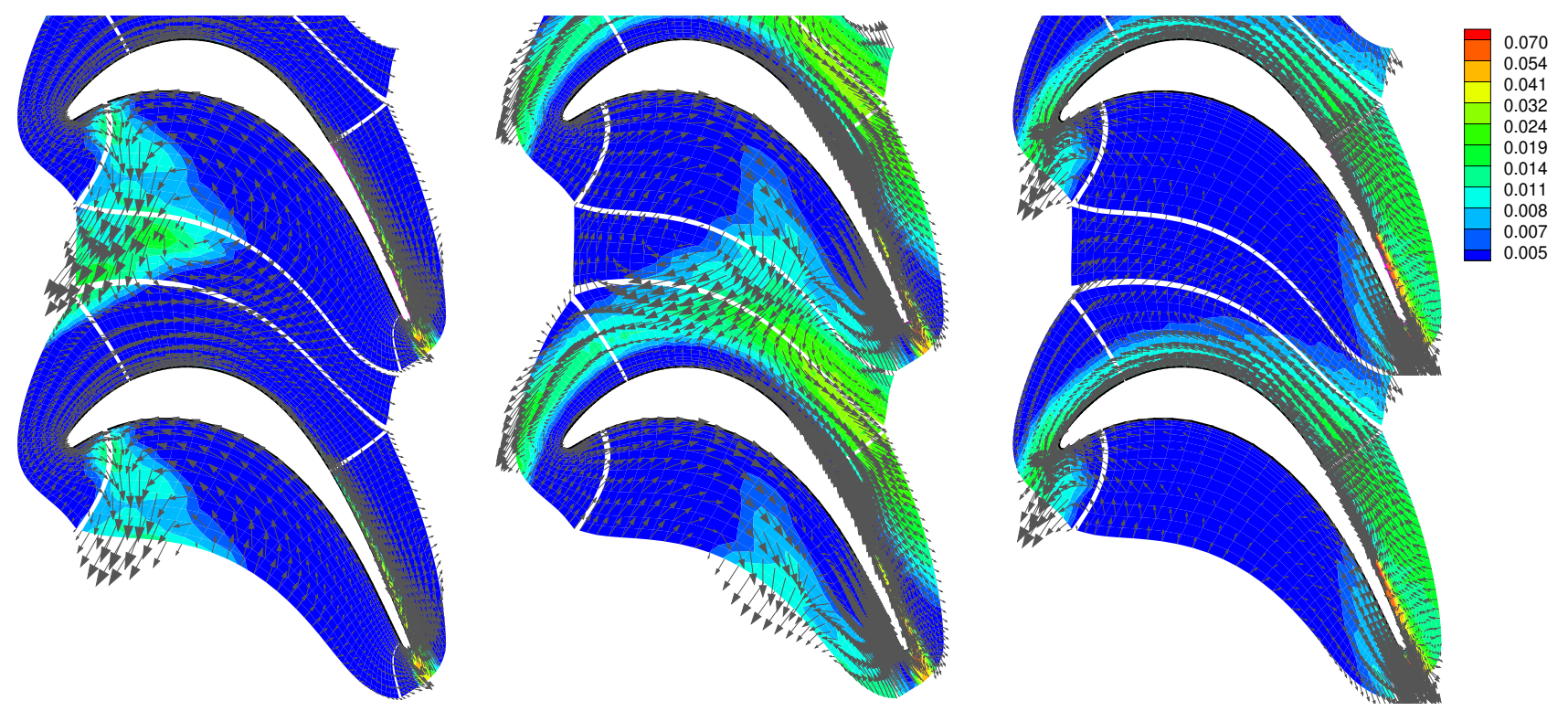

(a) 1B1U case
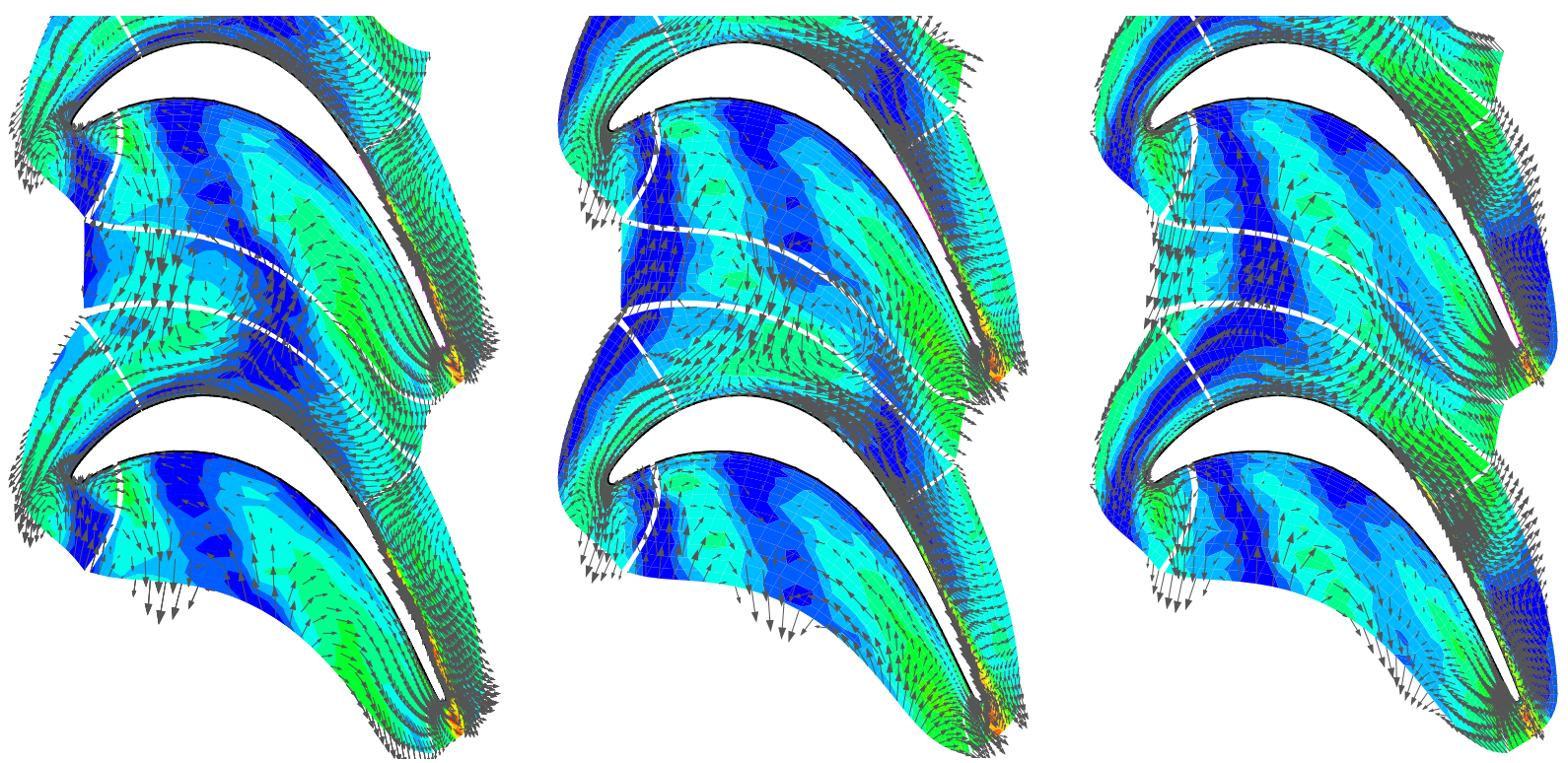

(b) $1 \mathrm{~B} 3 \mathrm{U}$ case

Fig. 2: CONTOUR PLOT OF PHASE-AVERAGED TKE AND VECTORIAL REPRESENTATION OF PHASEAVERAGED PERTURBATION VELOCITY FOR THREE TIME SNAPSHOTS. 


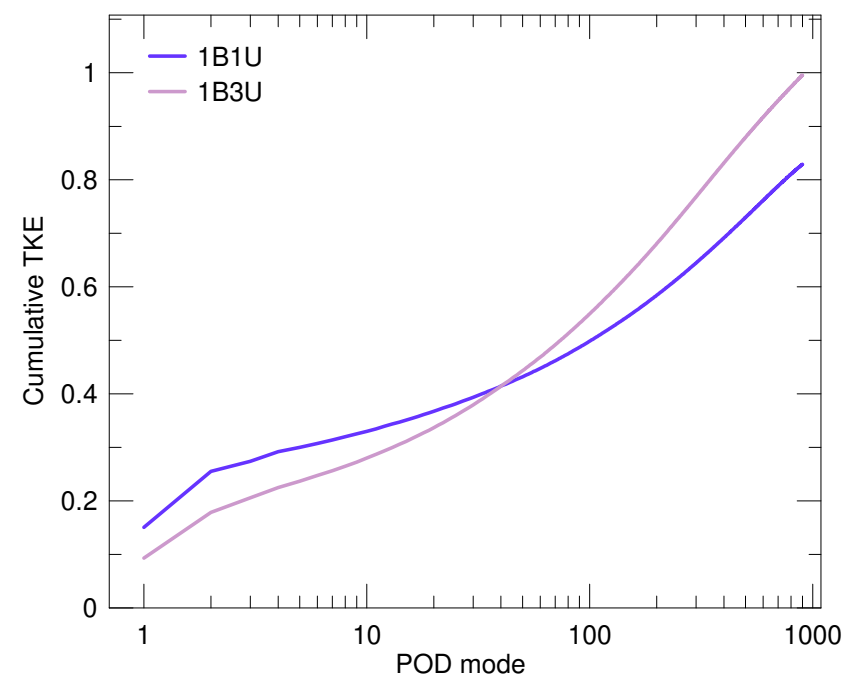

Fig. 3: CUMULATIVE CONTRIBUTION TO TKE.

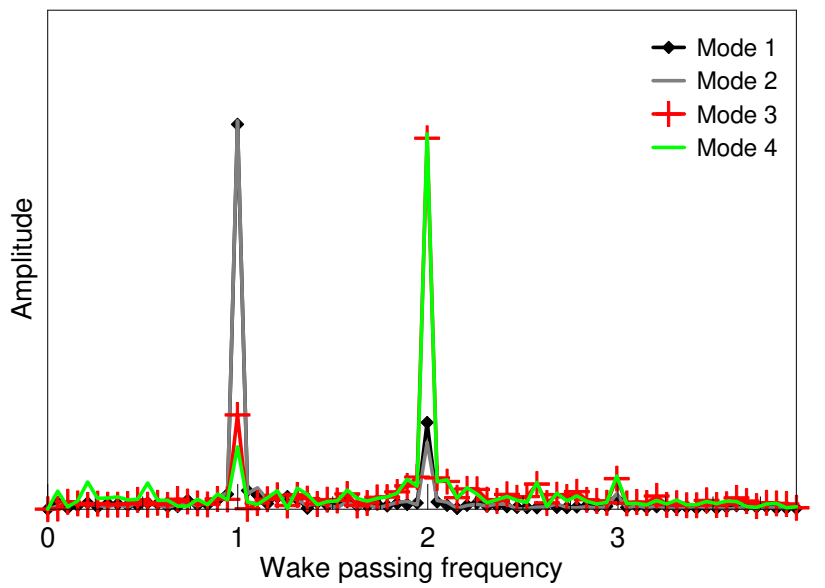

(a) $1 \mathrm{~B} 1 \mathrm{U}$ case

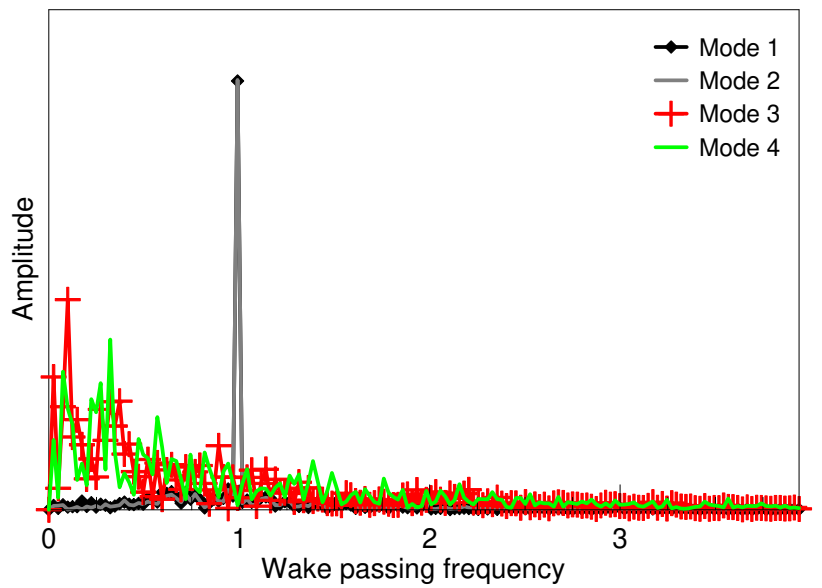

(b) 1B3U case

Fig. 4: SPECTRAL ANALYSIS OF THE POD EIGENVECTORS. 

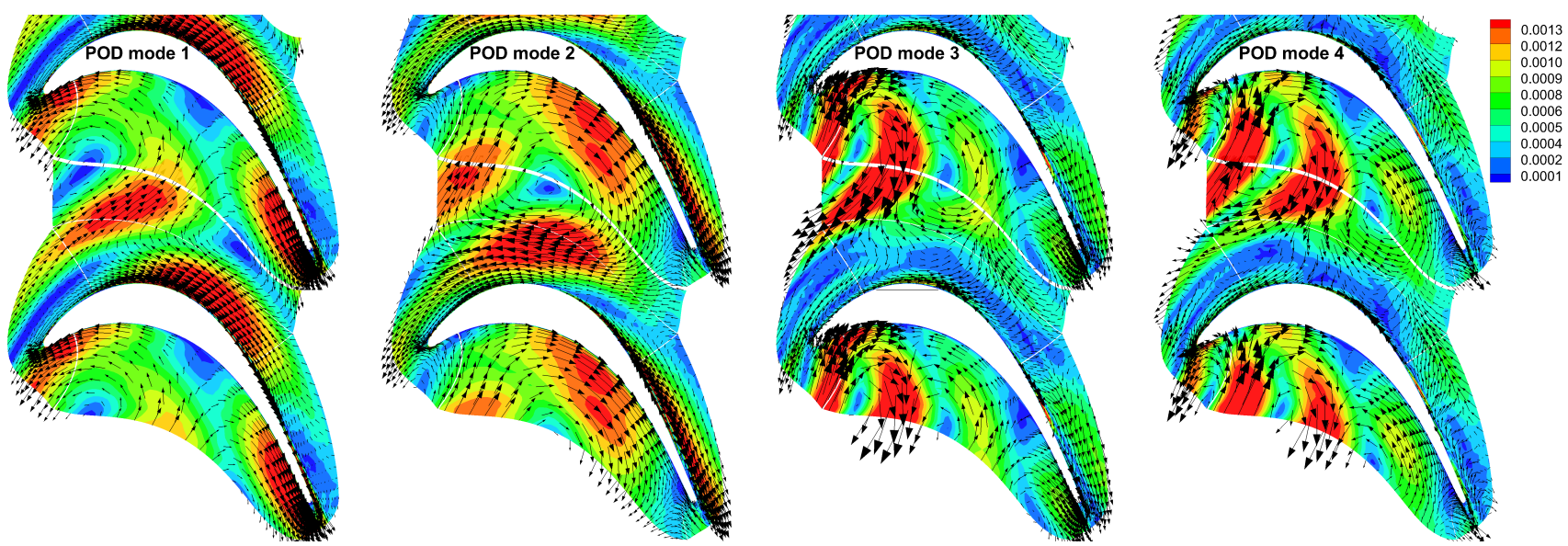

(a) $1 \mathrm{~B} 1 \mathrm{U}$ case
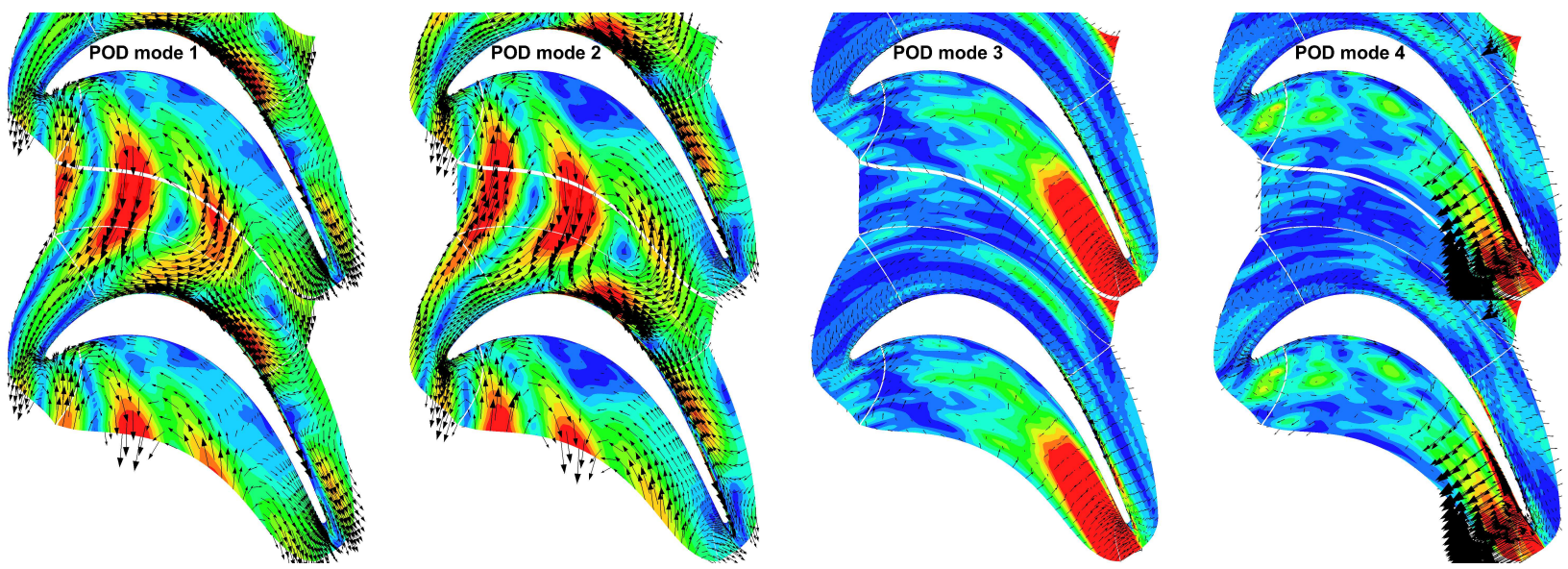

(b) $1 \mathrm{~B} 3 \mathrm{U}$ case

Fig. 5: CONTOUR PLOT OF TKE AND VECTORIAL REPRESENTATION OF POD MODES. 

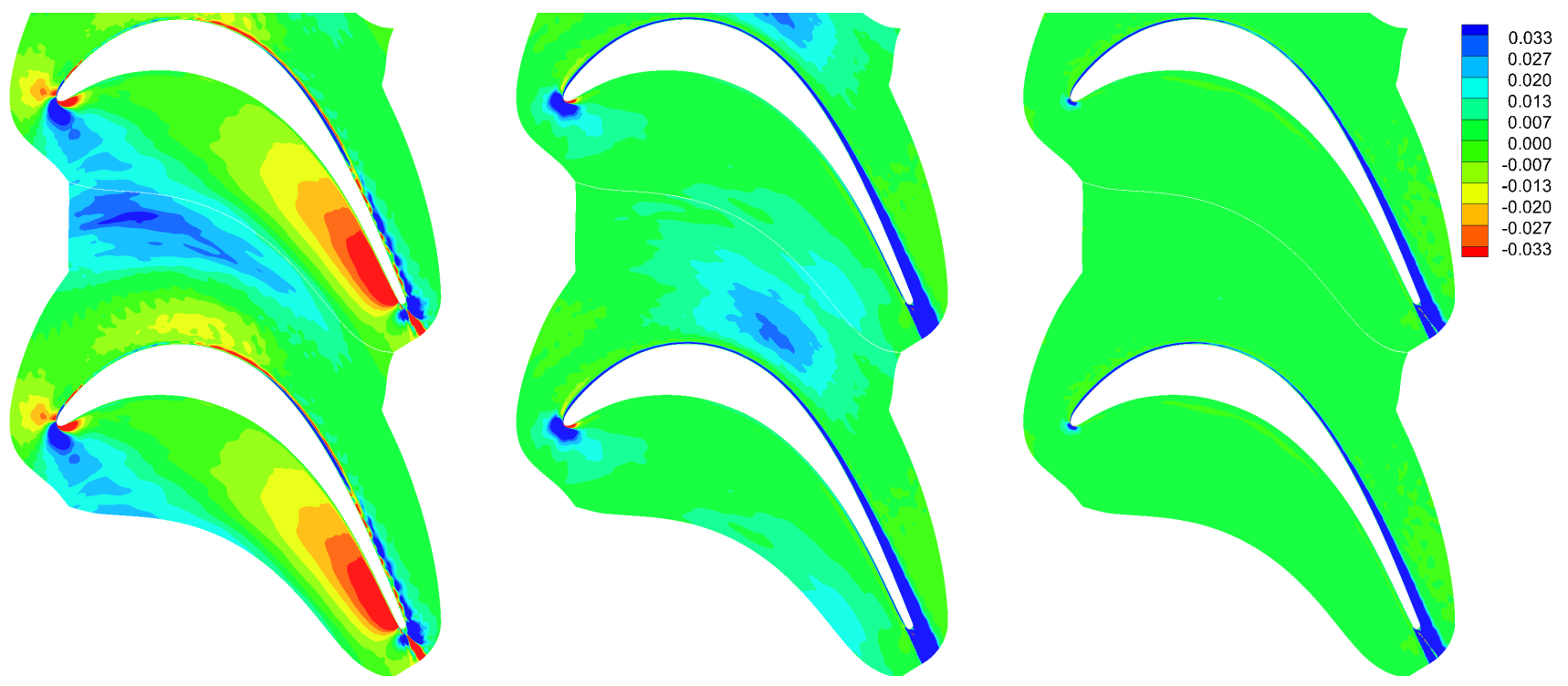

(a) 1B1U case
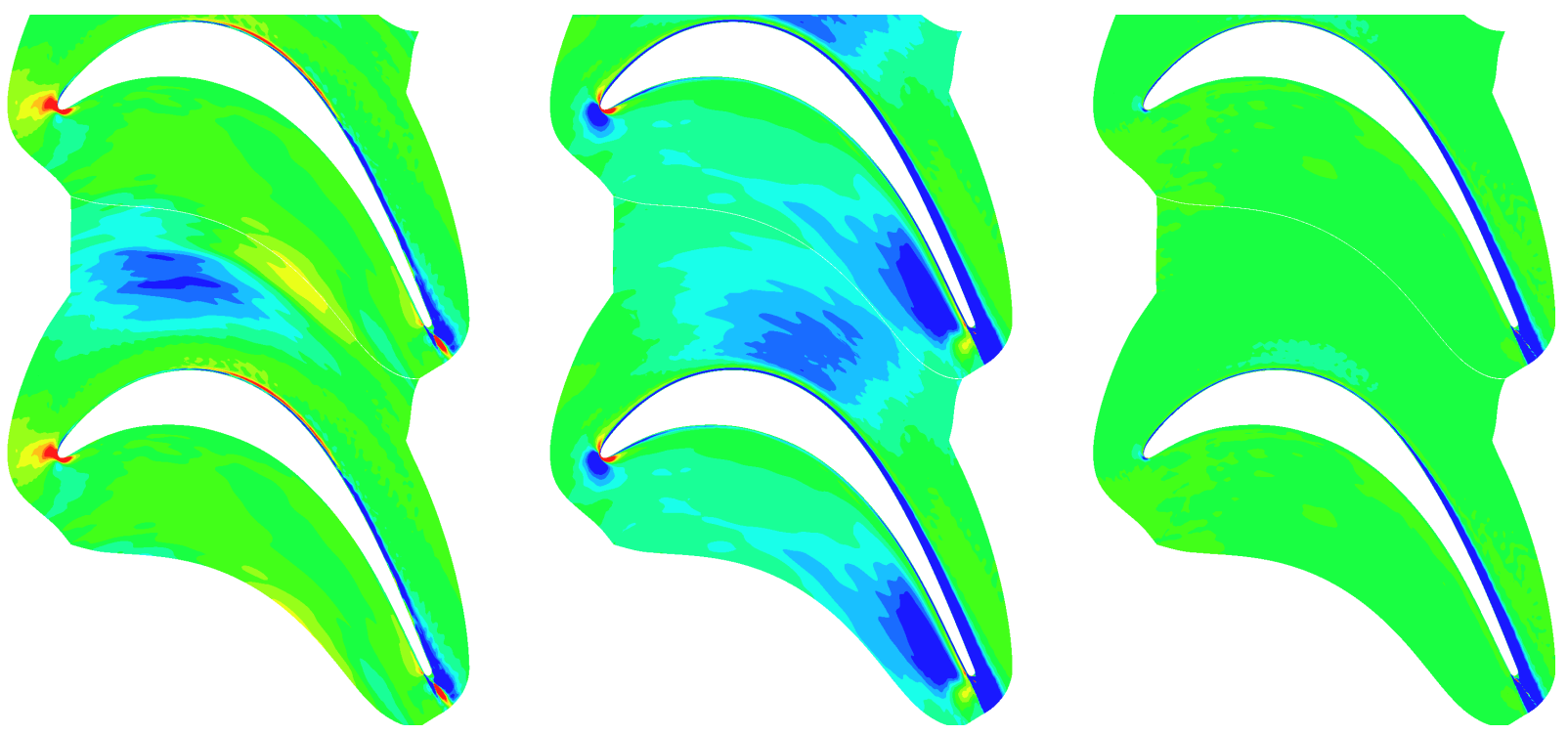

(b) $1 \mathrm{~B} 3 \mathrm{U}$ case

Fig. 6: CUMULATIVE CONTRIBUTION TO THE PRODUCTION OF TKE OF DIFFERENT RANGES OF POD MODES: MODES RELATED TO THE DETERMINISTIC PART OF THE PASSING WAKE (LEFT COLUMN), MODES RELATED TO THE TURBULENCE CARRIED BY THE WAKES AND ITS INTERACTION WITH THE BOUNDARY LAYER (MID COLUMN), MODES RELATED TO THE FINER SCALE STRUCTURES (RIGHT COLUMN). 

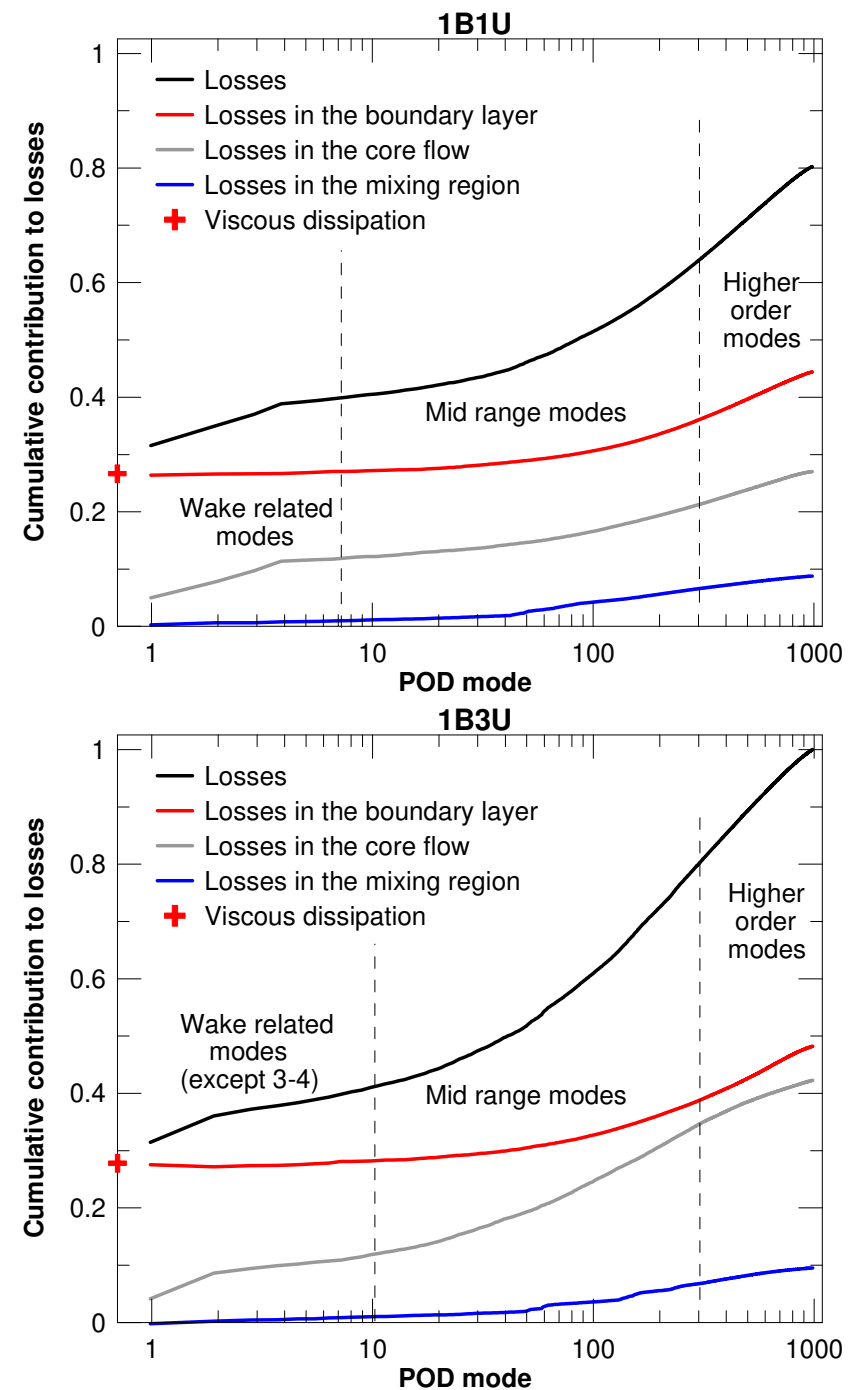

Fig. 7: CUMULATIVE CONTRIBUTION TO THE LOSSES (ENTROPY RATE OF CHANGE) OF EACH POD MODE. THE VALUES ARE OBTAINED BY INTEGRATION OVER THE 3D MEASUREMENT DOMAIN. THE PLOTS ARE NORMALIZED BY THE TOTAL LOSSES OF THE 1B3U CASE. 

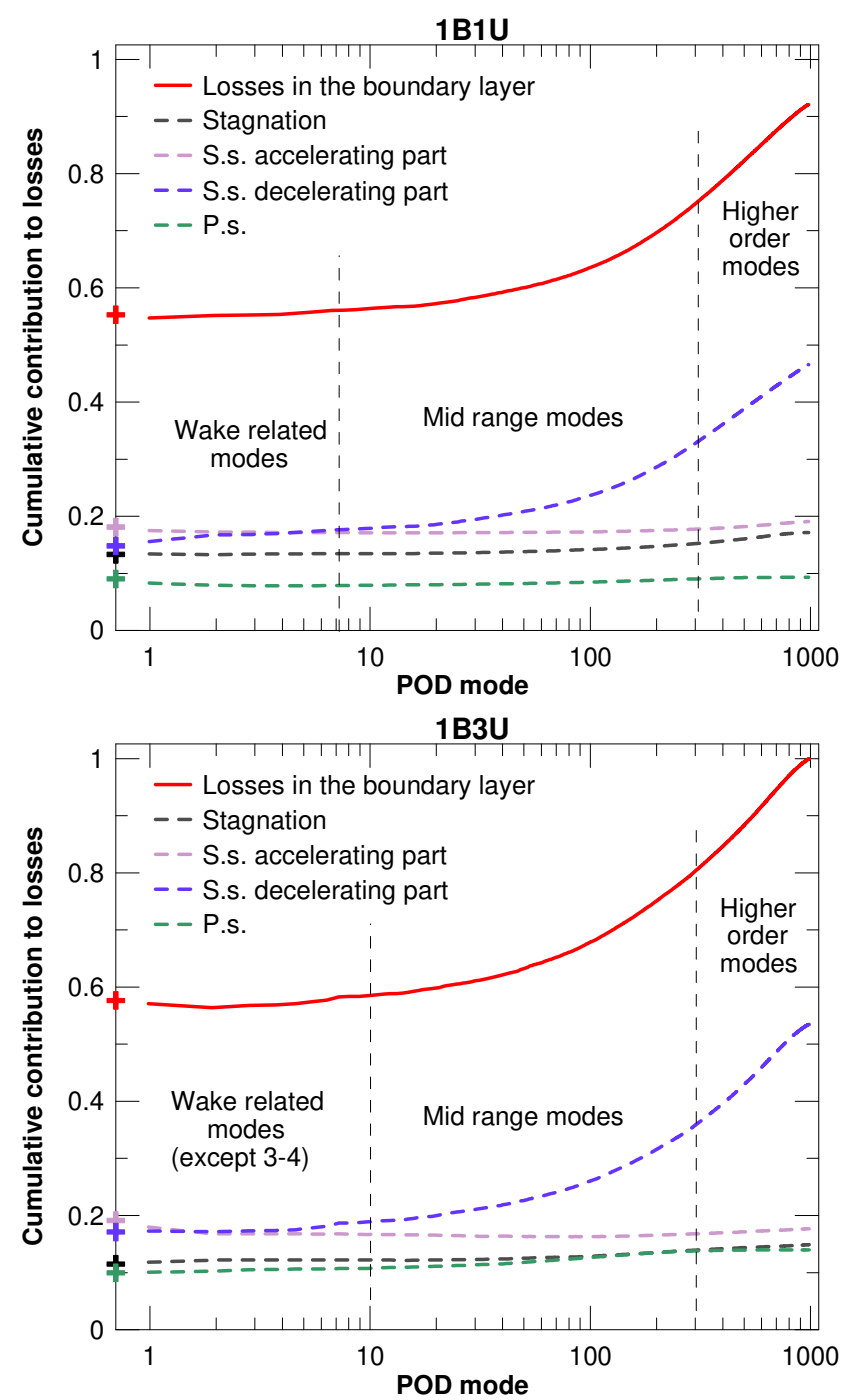

Fig. 8: CUMULATIVE CONTRIBUTION TO THE LOSSES IN THE BOUNDARY LAYERS OF EACH POD MODE. THE VALUES ARE OBTAINED BY INTEGRATION OVER THE 3D MEASUREMENT DOMAIN. THE PLOTS ARE NORMALIZED BY THE BOUNDARY LAYER LOSSES OF THE 1B3U CASE. SYMBOLS INDICATE THE VISCOUS DISSIPATION CONTRIBUTION 


\section{University Library}

\section{- M M N E R VA A gateway to Melbourne's research publications}

Minerva Access is the Institutional Repository of The University of Melbourne

Author/s:

Lengani, D;Simoni, D;Pichler, R;Sandberg, R;Michelassi, V;Bertini, F

Title:

On the Identification and Decomposition of the Unsteady Losses in a Turbine Cascade

Date:

2018-01-01

Citation:

Lengani, D., Simoni, D., Pichler, R., Sandberg, R., Michelassi, V. \& Bertini, F. (2018). On the Identification and Decomposition of the Unsteady Losses in a Turbine Cascade. Proceedings of the ASME Turbo Expo: Turbomachinery Technical Conference and Exposition, 2018, VOL 2A, 2A-2018, American Society of Mechanical Engineers. https:// doi.org/10.1115/GT2018-75724.

Persistent Link:

http://hdl.handle.net/11343/241879 